\title{
Incidence and risk factors for nerve injuries in mandibular third molar surgery
}

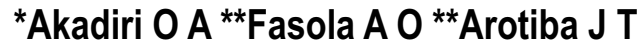 \\ *Department of Oral \& Maxillofacial Surgery, Faculty of Dentistry, College of Health Sciences, University of Port- \\ Harcourt, PortHarcourt, Rivers State, PMB 1, Port-Harcourt **Department of Oral \& Maxillofacial Surgery, \\ University College Hospital, Ibadan, PMB 5116, Ibadan
}

\begin{abstract}
Background: The inferior alveolar (IAN), lingual (LN) and long buccal nerves (LBN)are the three terminal branches of the trigeminal nerve which are susceptible to injury during surgical extraction of impacted mandibular third molars. While it is not always possible to accurately predict the patients that will be affected with these complications, understanding and identifying the risk factors may allow the adoption of appropriate technique and expertise for specific cases. We embarked on this study to document the incidence and duration of injury to the inferior alveolar nerve (IAN), lingual nerve ( $L N)$ and long buccal nerve ( $L B N)$ following the operative removal of impacted mandibular third molars and to identify the associated radiographic and operative risk factors.

Method: Pre- and postoperative neurosensory tests were performed for seventy nine patients who had surgical extraction of unilateral impacted mandibular third molars to determine the incidence and duration of complicating nerve injuries. The risk factors for nerve injury were determined among the radiographic variables and documented operative events.
\end{abstract}

Results: The incidence reported were $6.6 \%$ for IAN, $2.6 \%$ for $L N$ and $4.0 \%$ for $L B N$; all but one of the nerve injuries resolved within 2 weeks. Depth of impaction (Pell\& Gregory Level C) and linguo-version were the significant risk factors for IAN and LN injuries respectively while no risk factors was detected for LBN injury. Some significant operative events were associated with nerve injuries

Conclusion: Nerve injury in third molar surgery can be predicted based on some radiographic risk factors and some unforeseen intraoperative events. Most of the injuries are transitory in nature.

Keywords: Nerve injury, Radiographic risk factors, operative risk factors, Third molar surgery

Date Accepted for publication: 18th July 2009
Nig J Med 2009;402 - 408
Copyright @ 2009 Nigerian Journal of Medicine

\section{Introduction}

The inferior alveolar, lingual and long buccal nerves are the three terminal branches of the trigeminal nerve which are susceptible to injury during surgical extraction of impacted mandibular third molars ${ }^{1,2}$. The Injuries result in dysfunctional symptoms such as paraethesia, anaesthesia and dysaethesia, the management of which could be very complex and sometimes unsatisfactory; resulting in permanent deterioration in quality of life ${ }^{3,4}$. Therefore, it is imperative that this operative procedure be undertaken with reasonable caution and professional prowess.

Though reported to be generally rare, the documented incidence of nerve injury following impacted mandibular third molar extraction ranges from $0.4 \%$ to $5 \%$ for the inferior alveolar nerve (IAN); $0.6 \%=2 \%$ for lingual nerve (LN) and $0-0.27 \%$ for long buccal nerve ${ }^{5-9}$. These include cases performed by individuals of varying competence, using different techniques, under different settings and involving patients of diverse race and ethnicity. In our search of the literature, we observed a dearth of information on the incidence of nerve injuries associated with impacted third molar extractions in Nigeria.

While it is not always possible to accurately predict the patients that will be affected with these complications, understanding and identifying the risk factors may allow the adoption of appropriate technique and expertise for specific cases. Although some of the risk factors are observed intraoperative, majority can be easily identified during clinical and radiographic examinations. Although panoramic radiography is the recommended technique, sophisticated techniques such as, magnetic resonance imaging (MRI), tuned aperture computerized tomography (TACT), volumetric computerized tomography (VCT) and three dimensional reconstruction computerized tomography (3-D CT) have greatly enhanced the recognition of many of these risk factors in the developed world ${ }^{10,11,12}$. Apart from the limited or non-availabilty of some of these facilities, it is really impracticable to routinely request these 
expensive and technically demanding investigations for dental extractions in our environment. Consequently, radiographic assessment for patients at the risk of this complication in Nigeria has largely relied on the periapical radiography which has a lot of limitations and shortcomings. Although a few private-owned facilities in Nigeria now have equipment for panoramic radiography, this is similarly fraught with shortcomings.

We embarked on this study to document the incidence and duration of injury to the inferior alveolar nerve (IAN), lingual nerve (LN) and long buccal nerve (LBN) following the operative removal of impacted mandibular third molars and to identify the associated radiographic and operative risk factors. We discussed our observations in the light of available information in the world literature.

\section{Patients and Method}

The protocol for the study was approved by the Institutional Review / Local Ethics Committee and every participating patient gave an informed consent. Over a 12-month period 79 patients consented to participate in the study out of 102 patients who had impacted mandibular third molar extraction in our oral surgery clinic during the period. The participants were comprehensively examined and underwent complete pre- and post-treatment clinical neurosensory test by a modification of the technique described by Pogrel ${ }^{13}$ and Miloro et $\mathrm{al}^{14}$. This was done to ensure that none of them had any clinical evidence of preoperative sensorineural deficiency of the inferior alveolar (IAN), lingual (LN) and long buccal nerves (LBN). The technique involved both objective and subjective tests. In the subjective test, the patients were asked to describe any change in sensation in the areas around the mouth and to compare with the unoperated side and the pre-op sensation at the operated side. They were to touch the spots where they perceived altered sensation which was documented by the examiner. The objective test, consisted of 3 components in our modification as opposed to the two described by the original authors. First, a cotton wool pledget was use to stroke the tested sites in a particular direction to assess for light touch and directional discrimination. Second, a pin-prick test was done to map out the areas of sensory loss and finally, a two-point discrimination test was performed using blunted points of a mathematical set divider.

Standard periapical radiographs of the impacted teeth were obtained and features which were previously implicated in the literature ${ }^{15}$ such as; angular orientation of the tooth (Mesioangular, Horizontal, Vertical,
Distoangular, Transverse), depth of impaction (Pell\& Gregory's Level A,B, or C), available arch space for the tooth (Pell \& Gregory's Class I, II, or III), relative horizontal tilt of the tooth (Erect, Bucco-version, Linguo-version), proximity to the mandibular canal (Distant or Close), periodontal space (Normal, widened, obliterated), number of root (Fused, two, three), root favourability (favourable, unfavourable) were documented. The same surgical protocol was used for the extraction which involved the elevation of a three-sided buccal flap for access, the crestal periodontal fibres on the lingual side were carefully detached but no lingual flap was raised. Buccal guttering was performed using a rose-head surgical bur and after adequate ostectomy, the tooth was luxated using Coupeland's elevators Nos 1,2, and /or 3 . Crown sectioning was done using a surgical fissure bur when it was found necessary to effect tooth delivery. The extraction sockets were thoroughly irrigated, suctioned and swabbed dry to allow direct visualization for any exposed nerve or residual bony spicules. The flap was closed with 2 stiches of $3 / 0$ black silk sutures. Every intraoperative event the operator considered notable was recorded; however, no intraoperative observation warranted the abandonment of procedure or exclusion of any patient. All extractions were carried out by a single operator who was a senior resident in the department.

Postoperatively, verbal and written instructions were given to patients and prophylactic antibiotics( Oral Amoxycilin $500 \mathrm{mg} 3$ times daily for 5 days) and analgesics (Oral Piroxicam 20mg twice daily for 3 days) were administered. Any significant postoperative event was also documented. Patients were recalled for postoperative review on day 1 , day 7 and day 14 . Neurosensory examination was repeated for the inferior alveolar nerve, lingual nerve and long buccal nerve using the same protocol described above. Nerve function was coded normal or abnormal, when abnormal, it was documented as anaesthesia, paraesthesia.or dysaethesia

The incidence of injury to the IAN, LN and LBN were determined serially over the postoperative days 1,7 and 14. A multivariate regression of the radiographic variables was performed to determine the variables that constituted the most significant risk factors for injury to each of the nerves. Cases for which the operator documented significant intraoperative/postoperative events were specifically sorted out to find out if any of them had nerve injury probably resulting from the occurrence. 


\section{Results}

Seventy nine patients started the study of which four were excluded due to non compliance with follow up schedules. The four patients had turned up for review on postoperative day 1 but failed to show up subsequently. $A$ summary of the periapical radiographic records of those who completed the study are presented in Table I.

On post-op day 1 , the incidence of nerve injury observed were $6.6 \%$ (5 patients) for IAN, 2.6\% (2 patients) for LN and $4.0 \%$ (3 patients) for LBN. There was significant improvement on day 7 with the LBN and LN injury rate reducing to $1.3 \%$ (1 patient) each, while the incidence of IAN injury persisted as $6.6 \%$ (5 patients). On day 14 , there was complete recovery of IAN and LBN function while the incidence of LN injury persisted as 1.3\% (1 patient) [Fig. 1]. The alteration in nerve functions presented as paraesthesia in $4 / 5(80 \%)$ cases of IAN and $3 / 3(100 \%)$ of LBN injuries. One of five (20\%) IAN injuries and $2 / 2(100 \%)$ of $L N$ injuries presented as anaesthesia [Fig. 2].

To determine the risk factors for injury to each of the nerve, we performed a multivariate logistic regression using the radiographic variables in the models with injuries to each of the three nerves as the dependent variable. The significant predictors are depth of impaction [Level C] $\quad\left(P=0.034, X^{2}=12.037\right)$ for IAN injury and Horizontal tilt [Linguo-version] $\left(P=047, X^{2}=11.426\right)$ for $L N$ injury. On the other hand, no variable was significant for LBN injury.

When the documented intraoperative/postoperative events were reviewed with the incidence of nerve injury [Table II], it was noted that the patient with persistent lingual nerve anaesthesia had the lingual plate fused to the lingual surface of the root and was inadvertently fractured and luxated with the tooth. Notably, this patient had his impacted tooth in a linguo-version position. Also, $3 / 5$ of the patients with IAN injury had extensive and deeper than usual bone removal (in the experience of the operator) and crown sectioning associated with significant intraoperative bleeding. The other 2 patients with IAN injury experienced persistent prolonged brisk bleeding from the extraction socket greater than 20 minutes without extensive ostectomy. One patient had an electric shock feeling along the lips during IAN block and another one required repeated/intermittent administration of local anesthetic agent in spite of clinical evidence of effective anaesthesia. One patient returned to the emergency ward later for significant secondary hemorrhage from the operation site. None of the latter three patients had nerve injuries on postoperative review.

Table I. Summary of radiographic findings in the study populations

$\begin{array}{lc}\text { Depth of } & \text { Impaction } \\ \text { Level A: } & 51 \\ \text { Level B: } & 21 \\ \text { Level C: } & 2 \\ \text { Arch space } & \text { availability } \\ \text { Class 1: } & 19 \\ \text { Class 2: } & 53 \\ \text { Class 3: } & 3 \\ \text { Angular } & \\ \text { Mesientationgular: } & 25 \\ \text { Vertical: } & 20 \\ \text { Horizontal: } & 18 \\ \text { Distoangular: } & 12 \\ & \\ \text { Periodontal } & \text { space } \\ \text { Normal: } & 70 \\ \text { Widened: } & 5 \\ \text { Obliterated: } & 0\end{array}$

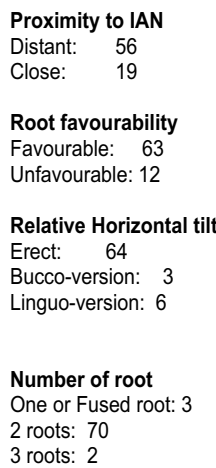

Table II. Association of notable operative events with nerve injury

\begin{tabular}{|lll|}
\hline $\begin{array}{l}\text { Intraoperative/postoperative events } \\
\text { related to nerve injury }\end{array}$ & & Outcome \\
\hline Fracture of lingual plate & - & Lingual nerve injury \\
Prolong brisk intra-alveolar bleeding (2 cases) & - & Inferior alveolar nerve injury \\
$\begin{array}{l}\text { Extensively deep ostectomy } \\
+ \text { coronectomy (3 cases) } \\
\text { Secondary heamorrrhage from socket }\end{array}$ & - & Inferior alveolar nerve injury \\
$\begin{array}{l}\text { Electric shock sensation during LA injection } \\
\begin{array}{l}\text { Persistent sensation of pain in spite of } \\
\text { clinical evidence of effective LA }\end{array}\end{array}$ & - & No nerve injury \\
\hline
\end{tabular}

Fig. 1. Incidence of nerve injury

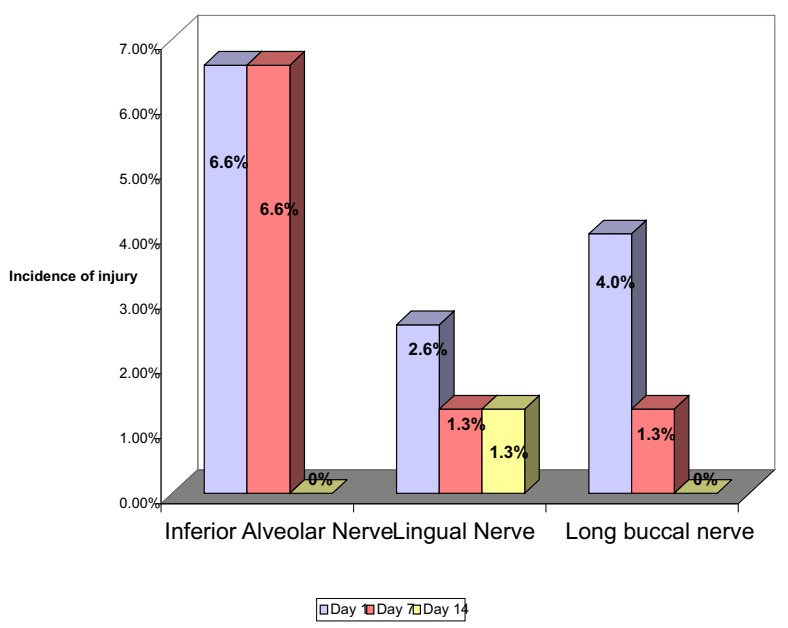

Fig. 2. Symptoms of nerve injury

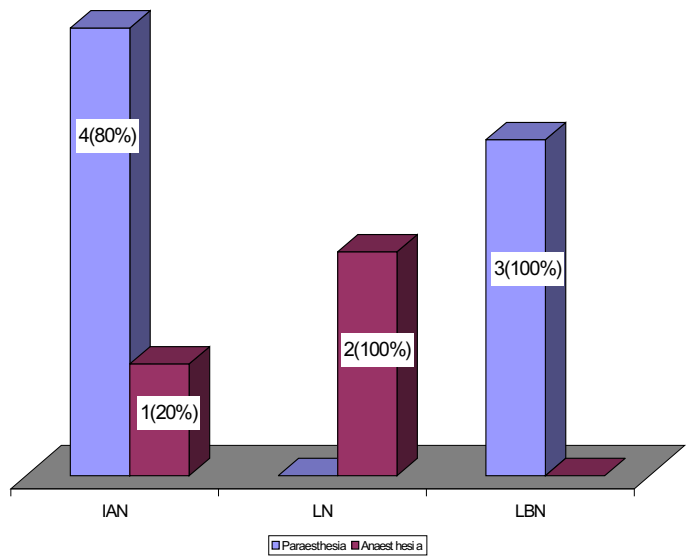




\section{Discussion}

Nerve injury involving the IAN, LN and LBN is a known neurologic complication of third molar surgery. In Nigeria, there is general paucity of reports on this complication. In the present study we observed the incidence and duration of nerve injury following impacted mandibular third molar surgery, more importantly, certain risk factors were identified.

The reported incidence for inferior alveolar nerve injury is $0.4 \%-5 \%, \frac{5,6,7}{}$. The incidence in this study being $6.6 \%$ falls within the reported range. In the first postoperative day, all the patients presented with paraethesia involving varying amount of area in the oral and perioral region including the lower lip, mentum and gingivae of the lower premolar/anterior teeth except one who had complete numbness around the lower lip. Although sensory abnormality in the oral region for any length of time is disturbing, it is much more agonizing when the symptoms persist as a permanent disability. Permanent sensory dysfunction is suspected when symptoms persists over 4 6 months without appreciable improvement ${ }^{18,19}$. None of the cases in this study persisted beyond 2 weeks resulting in zero percent incidence of permanent injury. The depth of impaction Pell \& Gregory's level $\mathrm{C}$ was the significant risk factor for IAN injury in this study. Although this is previously documented ${ }^{16}$, the radiographic factor most strongly associated in the literature is the proximity of the root apices to the mandibular canal ${ }^{12,20}$ which was not statistically significant in this study. This observation must be interpreted with caution considering the limitation of the 2-dimensional radiographic technique employed. When using 2-dimensional imaging modalities such as periapical or panoramic techniques, definite criteria ${ }^{21}$ have been described for determining the relationship between the roots of an impacted molar and the neurovascular bundle however, some cases are still inevitably misjudged because of the inability to appreciate the radiographic picture in three dimensions. This is the basis for suggesting 3-dimensional techniques such as 3-D CT scan and Volumentric Computerized Tomography (VCT) to confirm suspected cases ${ }^{11}$. These modalities allow the surgeon to achieve a realistic impression of the overall patho-anatomic situation preoperatively ${ }^{11}$. Using these advanced techniques in selected cases where the panoramic radiographs suggested a risk to the neurovascular bundles, Maegawa et $\mathrm{al}^{12}$ demonstrated that the bucco-lingual position of the canal relative to the roots is more important in determining the patients actually at risk inspite of proximity observed on two dimensional films. The mandibular canal could lie buccal, lingual or in-between the roots of the impacted molars.
They also noted that the absence/incompleteness of cortication around a mandibular canal exposes the nerve to greater risk and this was found to be more frequent with lingually placed canals and those between the roots. These are important impressions that could not have been appreciated on periapical radiographs and could account for the inability to clearly differentiate between the low risk and high risk cases when studies are based on 2-dimensional imaging techniques. Hence, a considerable number of roots thought to be close to the IAN and to probably predispose to injury in this study were not so found.

Most authors reported LN injury in third molar surgery accounting for $0.6 \%-2 \%$ of cases ${ }^{23}$, the incidence rate of $2.6 \%$ in this study compares with this. The lingual nerve provides general sensory supplies to the anterior twothird of the tongue, sublingual mucosa, floor of the mouth, and the mandibular lingual gingival. In addition, the special sensory supply for taste in the anterior twothird of the tongue is also carried by the nerve therefore; injury to the nerve could be very debilitating. Anatomical studies on patients ${ }^{22}$ and cadavers ${ }^{23,24}$ and also with the help of imaging technique such as magnetic resonance imaging (MRI) ${ }^{25}$ have identified various risk factors that can cause injury to the lingual nerve in third molar surgery. These include six prominent factors ${ }^{26}: 1$. Close proximity or direct contact of the lingual nerve and the lingual plate in the third molar region, 2. Lingual flap retraction, 3. Linguo-version of the tooth, especially in distoangular impactions 4. Perforation in the lingual plate 5 . Difficulty of the procedure 6 . The operator's experience. Our finding in this study is that the linguoversion of the tooth was the predominant risk factor. Although it was impossible for us to study the preoperative position of the lingual nerve in relation to the lingual plate and the presence of lingual plate fenestration or dehiscence from a periapical radiograph, we neither palpate any bony deficiency in the lingual plate during clinical examination nor retract a lingual flap during the procedure. Hence, we do not think that any of the injuries resulted from these. However, it is not unlikely that the injured nerves were in close relationship with the lingual surface of the extracted tooth as reportedly found in many patients who have had chronic recurrent pericoronitis in whom resulting fibrosis often hold the nerve sheath bound around the alveolar crests $^{26}$.

The incidence of long buccal nerve injury recorded in this study is unusually high being $4.0 \%$ compare to a $0.27 \%$ previously reported from our centre. Although 
this complication is poorly reported, it is generally believed that the LBN is the least susceptible of the nerves and in the hand of an experienced operator; it is not expected to be so frequently injured. Previous studies have documented that comparatively low incidence of nerve injury occur with greater experience ${ }^{27}$. All the cases with LBN injury in this study presented as paraesthesia in the related buccal mucogingiva of the molar region which did not pose a major deterioration to the patients' quality of life, they all returned to normal by the second week after operation. Although no predisposing factor to LBN injury was identified in the study, the incision process, blunt trauma arising from the placement of flap retractor and extent of retraction has been suggested as a possible risk factor ${ }^{17}$. One paper did note long buccal involvement when the anatomical position was aberrant, i.e., coming off the IAN once it was already in the canal and coming out through a separate foramen on the buccal side of the mandible ${ }^{28}$.

Apart from radiographic factors, some operative variables such as surgical techniques, anaesthetic technique, surgeon experience, extensive ostectomy especially distal, crown sectioning, pain during root luxation, bleeding, exposure of nerves, primary closure of wound, and postoperative ecchymosis have been implicated $^{20}$. We documented significant intra- and postoperative events that could possibly result in complications and correlated these with cases of nerve injuries observed. Deep and extensive ostectomy could have resulted in a breach of the lamina dura of the mandibular canal thus exposing the nerves to injury during coronectomy or luxation with dental elevators. This could account for the case of three patients with IAN injury who had extensive ostectomy and bleeding from the socket during surgery. Extensive ostectomy is sometimes required for certain deep impactions especially distoangular and where the root curvature is not favourable for easy delivery. In the same vein, two patients who had prolonged primary haemorrhage from the extraction site inspite of moderate bone removal turned out with IAN injury. Abnormally prolonged bleeding from an impacted molar extraction socket in a haemostatically normal patient is often associated with trauma to the inferior alveolar neurovascular bundle ${ }^{27}$ or a vessel in an aberrant retromolar foramen ${ }^{29}$. Such trauma affecting the nerve sheath may be responsible for the injury recorded postoperatively. Furthermore, one patient who experienced lingual nerve anaesthesia sustained lingual plate fracture during tooth luxation whereby the plate which was fused to the lingual aspect of the roots got dislodged with the tooth. It is not unlikely for the lingual nerve to have been traumatized in this experience. Although the anatomic position of the lingual nerve in relation to the retromolar and third molar region is variable $^{22,23,24}$, inadvertent fracture of the lingual plate constitute a potential risk for injury.

There were other significant events recorded; a patient experienced electric shock sensation along the lip during inferior alveolar nerve block yet had no nerve dysfunction during postoperative reviews. Such sensation is said to occur when the nerve fibers is inadvertently barbed against the bone during injection for anaesthesia; it has been reported to result in nerve dysfunction post anaesthesia ${ }^{18}$. Another patient experienced periodic pain that required intermittent reinjection with local anaesthesia. Many possible causes for ineffective local anaesthesia are known ${ }^{30}$, the most likely cause in this case is aberrant anatomy of the IAN and the mandibular canal considering that objective and subjective evidence of IAN anaesthesia was repeatedly elicited in the patient. The likelihood of a retromolar canal in this patient was entertained. The retromolar canal is a rare anatomic variation found in the retromolar triangle, a small triangular shaped region posterior to the third molar tooth in the mandible. It has been suggested that the content of this canal originate from the mandibular neurovascular bundle before it enters the mandibular canal in which case anaesthetic agent deposited close to the mandibular foramen may not affect the nerve content of the retromolar canal which has been noted to give an unusual supply to the pulp and periodontium of the third molar tooth ${ }^{30,31}$. Injury to this aberrant neurovascular bundle could result in altered sensation and abnormal bleeding from the extraction site. Another patient had significant secondary haemorrhage that required admission for monitoring at the emergency ward. Secondary haemorrhage in this patient is explainable by the resurgence of severe pain post anaesthesia resulting in elevated blood pressure and reopening of local microvasculature in the operation site. The bleeding was arrested by ensuring adequate analgesia and local measure which involved the use of an argotone pack in the extraction socket held in place with a horizontal mattress suture of the surrounding soft tissue.

In the majority of cases, altered sensation is a transitory phenomenon $^{20,33}$, when it persists beyond 6 months without any improvement the nerve is considered to be permanently injured ${ }^{18}$. All but one case of the injured nerves recovered completely within 2 weeks suggesting that most of the patients experienced 
neuropraxic type of injury. The only case of persistent lingual nerve anaesthesia happened in a patient who sustained lingual plate fracture during the extraction and who may have had significant trauma on the nerve resulting in neurotmesis or axonotemesis. This has different implications in the management of the injured patients. While temporary nerve dysfunction may respond to vitamin and steroid supplements, permanent nerve damage often require extensive and expensive treatments such as microneurosurgery and /or low level laser therapy ${ }^{4,19}$. In addition to being expensive, the facility and expertise for these modalities of treatment is not available in our environment. The only patient with persistent nerve damage in this study was lost to follow up and the final outcome of the management could not be determined.

\section{References}

1. 1. Gulicher D, Gerlach K.L. Sensory impairment of the lingual and inferior alveolar nerves following removal of impacted mandibular third molars. Int J Oral Maxillofac Sur. 2001; 30: 306312.

2. 2. Smith AC, Barry SE, Chiong AY et al. Inferior alveolar nerve damage following removal of mandibular third molar teeth. A prospective study using panoramic radiography. Aust Dent J 1997:42:149152

3. Martin SG, Michael G. Orofacaial pain: In Burket's Oral Medicine; Diagnosis and treatment $10^{\text {th }}$ ed. Spain, BC Decker 2003 Pp 307-342

4. Larry MW, Eber LLS. Considerations in nerve repairs. Proc (Bayl Univ Med Cent). 2003; 16: 152156

5. Chiapasco M, De Cicco L, Marrone G. Side effects and complications associated with third molar surgery. Oral Surg Oral Med Oral Pathol 1993;76:412420.

6. Middlehurst RJ, Barker GR, Rood JP. Postoperative morbidity with mandibular third molar surgery: a comparison of two techniques. J Oral Maxillofac Surg. 1988; 46: 474476

7. Sisk AL, Hammer WB, Shelton DW, Joy ED Jr. Complications following removal of impacted third molars: the role of the experience of the surgeon. J Oral Maxillofac Surg. 1986;44: 855859

8. 8. Lopes V, Mumenya R, Feinmann C, Harris M. Third molar surgery: an audit of the indications for surgery, post-operative complaints and patient satisfaction. Br J Oral Maxillofac Surg. 1995; 33: 3335.

9. Robinson PP, Smith KG. Lingual nerve damage during lower third molar removal: a comparison of two surgical methods. $\mathrm{Br}$ Dent J 1996; 180: 456-461.

10. Pawelzik J, Cohnen M, Willers R and Becker J. A comparison of conventional Panoramic radiographs with volumetric computed tomography images in the pre-operative assessment of impacted mandibular third molars. J Oral Maxillofac Surg 2000; 60:979-984.

11. Mozzo P, Procacci C, Tacconi A. A new volumetric CT machine for Dental imaging based on the cone-beam technique preliminary results. Eur Radiol 1998; 8:1558- 1562.

\section{Concluesion}

In this study we identified depth of impaction (Pell \& Gregory's level C), and linguo-version as the risk factors for IAN and LN injuries respectively. Certain important operative events such as extensive ostectomy and prolonged brisk bleeding from the socket also appear to be important factors associated with IAN injury while lingual plate fracture is associated with LN injury. No risk factor for LBN injury was discovered. The study supports the fact that nerve injury following third molar extraction is usually transitory in nature and patients often recover spontaneously and completely. The limitation associated with the use of 2-dimensional imaging for assessing impacted third molar was highlighted.

12. Maegawa $\mathrm{H}$, Sano K, Kitagawa $\mathrm{Y}$. Preoperative assessment of the relationship between the mandibular third molar and the mandibular canal by axial computed tomography with sagittal and coronal reconstruction. Oral Surg Oral Med Oral Pathol Oral Radiol Endod 2003; 96: 639-646.

13. Pogrel MA. The results of microneurosurgery of the inferior alveolar and lingual nerve. J Oral Maxillofac Surg. 2002; 60: 485489

14. Miloro M, Repasky M. Low-level laser effect on neurosensory recovery after sagittal ramus osteotomy. Oral Surg Oral Med Oral Pathol Oral Radiol Endod. 2000; 89: 1218.

15. Santamaria J, Arteagoitia I. Radiological Variables of clinical significance in the extraction of impacted third molars. Oral Surg Oral Med Oral Pathol Radiol Endod 1997; 84: 469-463.

16. Bui $\mathrm{CH}$, Seldin EB, Dodson TB. Types, frequency and risk factors for complication after third molars extraction. J Oral Maxillofac Surg 2003;61:1379-1389.

17. Obiechina A.E. Paraesthesia after mandibular third molar extractions in Nigerians. Odonto- stomalogie Tropicale 1991; 2: 113-115.

18. Pogrel MA, Thamby S. Permanent nerve involvement resulting from inferior alveolar nerve blocks. JADA 2000; 131: 9017.

19. Tuncer Ozen, Kaan Orhan, Ilker Gorur, Adnan Ozturk. Efficacy of low level laser therapy on neurosensory recovery after injury to the inferior alveolar nerve. Head Face Med. 2006; $2: 3-8$

20. Eduard Valmaseda-Castellon, Leonardon Berini-Aytes, Cosme Gay-Escado. Inferior Alveolar Nerve damage after third molar surgical extractions. A prospective study of 1117 surgical extractions. Oral Surg Oral Med Oral Pathol Oral Radiol Endod 2001:92: 377-383.

21. Howe GL, Poyton HG. Prevention and damage to the inferior dental nerve during the extraction of mandibular third molar. BrDent J 1960; 109:355-363.

22. Kiesselbach JE, Chamberlain JG. Clinical and anatomical observations of the relationships between the lingual nerve and the mandibular third molar region. J Oral Maxillofac Surg 1984; 42: 565-567 
23. Pogrel MA, Renaut A, Schimidt B et al. The relationship of the lingual nerve to the mandibular third molar region an anatomic study. J Oral Maxillofac Surg 1995; 53: 1178-1181

24. Holzle FW, Wolff KD. Anatomic position of the lingual nerve in the mandibular third molar region with special consideration of atrophied mandibular crest: an anatomic study Int $\mathrm{J}$ Oral Maxillofac Surg 2001; 30; 333-338

25. Miloro M, Halkias LE, Slone HW et al. Assessment of the lingualnerve in the mandibular third molar region using magnetic resonance imaging. J Oral Maxillofac Surg 1997; 55: 134-137

26. Toltunov L. Lingual nerve vulnerability: risk analysis and case reports. Compendium 2007; 28: 28-32

27. Jerjes W, El-Maaytah M, Swinson B, Banu B, Upile T, D'Sa S, AlKhawalde M, Chaib B, Hopper C. Experience versus complication rate in third molar surgery. Head Face Med. 2006; 2: 14.

28. Singh S. Aberrant buccal nerve encountered at third molar surgery. Oral Surgery, Oral Medicine, Oral Pathology 1981; $52: 142$
29. Robinson PD, Pitt Ford TR, McDonald F. Local anaesthesia in Dentistry. Wright, Woburn, 2000

30. Benediktsdottir IS, Wenzel A, Petersen JK, Hintze $H$. Mandibular third molar removal: risk indicators for extended operation time, postoperative pain, and complications. Oral Surg Oral Med Oral Pathol Oral Radiol Endod. 2004;97:438 446

31. Tuncer N, Bilecegnolu B. Clinical and anatomical study of the retromolar foramen and canal. J Oral Maxillofac Surg 2006; 64; 1493-1497

32. Anderson LC, Kosinki PF, Mentag PJ. A review of the intraosseous course of the nerves of the mandible. J Oral Implantol 1991; 17:394

33. Robinson PP, Loescher AR, Yates JM, Smith KG. Current management of damage to the inferior alveolar and lingual nerves as a result of removal of third molars. $\mathrm{Br} \mathrm{J}$ Oral Maxillofac Surg 2004; 42: 285292. 